\title{
Some Classes of Co-Schwarzian Functions and Its Coefficient Inequality that Is Sharp
}

\author{
Gurmeet Singh and Misha Rani
}

\begin{abstract}
In our present work, we defined an inequality called Fekete - Szegö Inequality for functions $f(z)$ in the classes of starlike functions and convex functions along with subclasses of these classes.

Keywords - Bounded analytic functions and concept of subordination, Convex functions, Fekete-Szegö Inequality, Starlike functions.
\end{abstract}

\section{INTRODUCTION}

We deal with geometric function theory and Koebe [6] proved that Riemann Mapping theorem is the main pillar of this theory. From this theorem, a conjecture called Bieberbach conjecture was produced. This conjecture was given by L. Bieberbach but finally proved by Louis De Branges[1]. While tackling with this conjecture, an equality arises called Fekete-Szegö Inequality, which was given by M. Fekete and G. Szegö [3]. Till now many researchers have solved this inequality for various classes and subclasses of starlike functions, convex functions, close-to-convex functions and for many other functions. Now, here we establish this inequality for different classes and subclasses of starlike functions and convex functions.

Firstly, we define some fundamental classes.

A consists of the family of analytic functions $f$ with the normalization $f(0)=0, f^{\prime}(0)=1$ and having functions of the type $f(z)=z+\sum_{k=2}^{\infty} a_{k} z^{k}$;

$S$ be the family of functions $f$ normalized by $f(0)=0, f^{\prime}(0)=1$ where $f(z)=z+\sum_{k=2}^{\infty} a_{k} z^{k}$; univalent in the open disk: $E=\{z \in C:|z|<1\}$ and $S^{*}(\phi)$ and $\mathrm{C}(\phi)$ be the class of functions inf $\in S$ for which $\frac{z f^{\prime}(z)}{f(z)} \prec \phi(\mathrm{z})$ and $\frac{\left[z f^{\prime}(z)\right]^{\prime}}{[f(z)]^{\prime}} \prec \phi(z) ;$ this was introduced by Ma and Minda [10].

In this equation, “ $<$ " denotes subordination [which states that let $f(z)$ and $g(z)$ are two analytic functions, if there exists a Schwarzian function $w(z)$ (analytic in $E$ ) in such a way that $|w(z)|<1, w(0)=0$ and $f(z)=g(w(z))$ $; z \in E$, then the function $f(z)$ is subordinate to $g(z)$ and we write it as $f(z)<g(z)]$.

The concept of subordination was given by Lindelof [7]. Here, $\phi(z)$ is an analytic function with positive real part on $E$ which maps the unit disk $E$ onto a region starlike with respect to 1 as well as symmetric with respect to real axis, satisfying conditions $\phi(0)=0$ and $\phi^{\prime}(0)>0$ and Schwarzian function is an analytic function of the type $w(z)=\sum_{n=1}^{\infty} c_{n} z^{n}$ with conditions $w(0)=0$ and $|w(z)|<1$.

Miller, S.S., Mocanu, P.T. and Reade, M.O. [9] proved the conditions $\left|c_{l}\right| \leq 1,\left|c_{2}\right| \leq 1-\left|c_{1}\right|^{2}$ for the above defined bounded analytic functions.

The class defined below is denoted by $\operatorname{TK}[\alpha, \beta]$ and is a subclass of $K$, satisfying the conditionalong with some subclasses:

$$
(1-\alpha) \frac{f(z)}{z}+\alpha \frac{\left\{z f^{\prime}(z)+\beta z^{2} f^{\prime \prime}(z)\right\}^{\prime}}{\{f(z)\}^{\prime}} \prec \phi(\mathrm{z})
$$

\section{MAIN RESULT}

THEOREM-1:

Let $f(z) \in T K[\alpha, \beta]$ and $\phi(z)=\frac{1+w(z)}{1-w(z)} ; w(z)$ isa Schwarzian function, then:

Published on August 10, 2021.

Gurmeet Singh, Department of Mathematics, Khalsa College, Patiala- 147002, Punjab, India.

(e-mail: meetgur111@gmail.com)

Misha Rani, Research Fellow, Department of Mathematics, Punjabi University, Patiala, Punjab, India.

(e-mail: mishagargsamana ${ }^{\circledR}$ gmail.com) 


$$
\left|a_{3}-\mu a_{2}^{2}\right| \leq\left\{\begin{array}{c}
\frac{16 \alpha(1+2 \beta)+2[1+\alpha(1+4 \beta)]^{2}}{[1+\alpha(1+4 \beta)]^{2}(1+5 \alpha+18 \alpha \beta)}-\frac{4 \mu}{[1+\alpha(1+4 \beta)]^{2}} \\
\mu \leq \frac{4 \alpha(1+2 \beta)}{1+5 \alpha+18 \alpha \beta} \\
\frac{2}{1+5 \alpha+18 \alpha \beta} ; \frac{4 \alpha(1+2 \beta)}{1+5 \alpha+18 \alpha \beta} \leq \mu \\
\leq \frac{4 \alpha(1+2 \beta)+[1+\alpha(1+4 \beta)]^{2}}{(1+5 \alpha+18 \alpha \beta)} \\
\frac{4 \mu}{[1+\alpha(1+4 \beta)]^{2}}-\frac{16 \alpha(1+2 \beta)+2[1+\alpha(1+4 \beta)]^{2}}{[1+\alpha(1+4 \beta)]^{2}(1+5 \alpha+18 \alpha \beta)} \\
\mu \geq \frac{4 \alpha(1+2 \beta)+[1+\alpha(1+4 \beta)]^{2}}{(1+5 \alpha+18 \alpha \beta)}
\end{array}\right.
$$

The result is sharp.

\section{PROOF:}

By definition of TK $[\alpha, \beta]$,

$$
(1-\alpha) \frac{f(z)}{z}+\alpha \frac{\left\{z f^{\prime}(z)+\beta z^{2} f^{\prime \prime}(z)\right\}^{\prime}}{\{f(z)\}^{\prime}}=\frac{1+w(z)}{1-w(z)}
$$

where

$$
\begin{gathered}
w(z)=c_{1} z+c_{2} z^{2}+c_{3} z^{3} \\
f(z)=z+a_{2} z^{2}+a_{3} z^{3} \\
f^{\prime}(z)=1+2 a_{2} z+3 a_{3} z^{2}+4 a_{4} z^{3} \\
f^{\prime \prime}(z)=2 a_{2}+6 a_{3} z+12 a_{4} z^{2}
\end{gathered}
$$

Now by putting all these values in (1), we get:

$$
(1-\alpha)\left(1+a_{2} z+a_{3} z^{2}+-\right) \alpha\left(\frac{\left\{z+2 a_{2} z^{2}+3 a_{3} z^{3}+\beta 2 a_{2} z^{2}+\beta 6 a_{3} z^{3}+\beta 12 a_{4} z^{4}+--------\right\}^{\prime}}{\left(1+2 a_{2} z+3 a_{3} z^{2}+----\right)}\right)=\frac{1+c_{1} z+c_{2} z^{2}+---}{1-\left(c_{1} z+c_{2} z^{2}+---\right)}
$$

By expanding the series, we get:

$$
\begin{gathered}
1+[1+\alpha(1+4 \beta)] a_{2} z+\left[(1+5 \alpha+18 \alpha \beta) a_{3}-4 \alpha(1+2 \beta) a_{2}^{2}\right] z^{2} \\
=1+2 c_{1} z+2\left(c_{2}+c_{1}^{2}\right) z^{2}
\end{gathered}
$$

By comparing, we get:

$$
\begin{gathered}
a_{2}=\frac{2 c_{1}}{1+\alpha(1+4 \beta)} \\
a_{3}=\frac{2[1+\alpha(1+4 \beta)]^{2} c_{2}+\left[16 \alpha(1+2 \beta)+2\{1+\alpha(1+4 \beta)\}^{2}\right] c_{1}^{2}}{(1+5 \alpha+18 \alpha \beta)\{1+\alpha(1+4 \beta)\}^{2}}
\end{gathered}
$$

Using these values of $a_{2}$ and $a_{3}$, we get:

$$
a_{3}-\mu a_{2}^{2}=\frac{2 c_{2}}{1+5 \alpha+18 \alpha \beta}+\left(\frac{16 \alpha(1+2 \beta)+2\{1+\alpha(1+4 \beta)\}^{2}}{\left.(1+5 \alpha+18 \alpha \beta)\{1+\alpha(1+4 \beta)\}^{2}\right)}-\frac{4 \mu}{\{1+\alpha(1+4 \beta)\}^{2}}\right) c_{1}^{2}
$$

After applying mode on both sides, we get: 


$$
\left|a_{3}-\mu a_{2}^{2}\right| \leq\left(\frac{2}{1+5 \alpha+18 \alpha \beta}\right)\left|c_{2}\right|+\left|\frac{16 \alpha(1+2 \beta)+2\{1+\alpha(1+4 \beta)\}^{2}}{(1+5 \alpha+18 \alpha \beta)\{1+\alpha(1+4 \beta)\}^{2}}-\frac{4 \mu}{\{1+\alpha(1+4 \beta)\}^{2}}\right|\left|c_{1}\right|^{2}
$$

Using $\left|c_{2}\right| \leq 1-\left|c_{1}\right|^{2}$, we get:

$$
\left|a_{3}-\mu a_{2}^{2}\right| \leq \frac{2}{1+5 \alpha+18 \alpha \beta}+\left\{\left|\frac{16 \alpha(1+2 \beta)+2\{1+\alpha(1+4 \beta)\}^{2}}{(1+5 \alpha+18 \alpha \beta)\{1+\alpha(1+4 \beta)\}^{2}}-\frac{4 \mu}{\{1+\alpha(1+4 \beta)\}^{2}}\right|-\frac{2}{1+5 \alpha+18 \alpha \beta}\right\}\left|c_{1}\right|^{2}
$$

\section{Case 1:}

$$
\mu \leq \frac{8 \alpha(1+2 \beta)+\{1+\alpha(1+4 \beta)\}^{2}}{2(1+5 \alpha+18 \alpha \beta)}
$$

Then

$$
\left|a_{3}-\mu a_{2}^{2}\right| \leq \frac{2}{1+5 \alpha+18 \alpha \beta}+\left|\frac{16 \alpha(1+2 \beta)}{(1+5 \alpha+18 \alpha \beta)\{1+\alpha(1+4 \beta)\}^{2}}-\frac{4 \mu}{\{1+\alpha(1+4 \beta)\}^{2}}\right|\left|c_{1}\right|^{2}
$$

Subcase - 1 (a):

$$
\mu \leq \frac{4 \alpha(1+2 \beta)}{1+5 \alpha+18 \alpha \beta}
$$

Using $\left|c_{1}\right| \leq 1$, we get:

$$
\left|a_{3}-\mu a_{2}^{2}\right| \leq \frac{2\{1+\alpha(1+4 \beta)\}^{2}+16 \alpha(1+2 \beta)}{(1+5 \alpha+18 \alpha \beta)\{1+\alpha(1+4 \beta)\}^{2}}-\frac{4 \mu}{\{1+\alpha(1+4 \beta)\}^{2}}
$$

Subcase - 1 (b):

$$
\mu \geq \frac{4 \alpha(1+2 \beta)}{1+5 \alpha+18 \alpha \beta}
$$

Then

$$
\left|a_{3}-\mu a_{2}^{2}\right| \leq \frac{2}{1+5 \alpha+18 \alpha \beta}
$$

Case - 2:

$$
\mu \geq \frac{8 \alpha(1+2 \beta)+\{1+\alpha(1+4 \beta)\}^{2}}{2(1+5 \alpha+18 \alpha \beta)}
$$

Then

$$
\left|a_{3}-\mu a_{2}^{2}\right| \leq \frac{2}{1+5 \alpha+18 \alpha \beta}+\left|\frac{4 \mu}{\{1+\alpha(1+4 \beta)\}^{2}}-\frac{16 \alpha(1+2 \beta)+4\{1+\alpha(1+4 \beta)\}^{2}}{(1+5 \alpha+18 \alpha \beta)\{1+\alpha(1+4 \beta)\}^{2}}\right|\left|c_{1}\right|^{2}
$$

Subcase-2 (a):

$$
\begin{gathered}
\mu \geq \frac{4 \alpha(1+2 \beta)+\{1+\alpha(1+4 \beta)\}^{2}}{(1+5 \alpha+18 \alpha \beta)} \\
\text { Then }\left|a_{3}-\mu a_{2}^{2}\right| \leq-\frac{2\{1+\alpha(1+4 \beta)\}^{2}+16 \alpha(1+2 \beta)}{(1+5 \alpha+18 \alpha \beta)\{1+\alpha(1+4 \beta)\}^{2}}+\frac{4 \mu}{\{1+\alpha(1+4 \beta)\}^{2}}
\end{gathered}
$$

Subcase - 2 (b):

$$
\mu \leq \frac{4 \alpha(1+2 \beta)+\{1+\alpha(1+4 \beta)\}^{2}}{(1+5 \alpha+18 \alpha \beta)}
$$


Then

$$
\left|a_{3}-\mu a_{2}^{2}\right| \leq \frac{2}{1+5 \alpha+18 \alpha \beta}
$$

Combining (2), (3), (4) and (5), we get the required result.

Extremal functions of this inequality are given by:

$$
f(z)=\mathrm{z}\left(1-\frac{2 \alpha[(\alpha+5)+2 \beta\{3+4 \alpha(1+2 \beta)\}]}{(1+\alpha+4 \alpha \beta)(1+5 \alpha+18 \alpha \beta)} z\right)^{-\frac{1+5 \alpha+18 \alpha \beta}{\alpha(\alpha+5)+2 \beta\{3+4 \alpha(1+2 \beta)\}}}
$$

and $f(z)=\mathrm{z}\left(1+2 z^{2}\right)^{\frac{1}{1+5 \alpha+18 \alpha \beta}}$.

COROLLARY-2:

$T K[1,0]=K$, as by substituting $\alpha=1$ and $\beta=0$ the result becomes:

$$
\left|a_{3}-\mu a_{2}^{2}\right| \leq\left\{\begin{array}{l}
1-\mu, \text { if } \mu \leq \frac{2}{3} \\
\frac{1}{3}, \text { if } \frac{2}{3} \leq \mu \leq \frac{4}{3} \\
\mu-1, \text { if } \mu \geq \frac{4}{3}
\end{array}\right.
$$

which is the required result for the class $K$ given by Keogh and Merkes [5].

\section{THEOREM3:}

Let $f(z) \in T K[\alpha, \beta, \delta]$ and $\phi(z)=\frac{1+w(z)}{1-w(z)} ; w(z)$ is a Schwarzian function, then:

$$
\left|a_{3}-\mu a_{2}^{2}\right| \leq\left\{\begin{array}{c}
\frac{16 \alpha(1+2 \beta) \delta^{2}+2 \delta^{2}[1+\alpha(1+4 \beta)]^{2}}{[1+\alpha(1+4 \beta)]^{2}(1+5 \alpha+18 \alpha \beta)}-\frac{4 \mu \delta^{2}}{[1+\alpha(1+4 \beta)]^{2}} \\
\mu \leq \frac{8 \alpha(1+2 \beta) \delta^{2}+\left(\delta^{2}-\delta\right)[1+\alpha(1+4 \beta)]^{2}}{1+5 \alpha+18 \alpha \beta} ; \\
\frac{2 \delta}{1+5 \alpha+18 \alpha \beta} ; \frac{8 \alpha(1+2 \beta) \delta^{2}+\left(\delta^{2}-\delta\right)[1+\alpha(1+4 \beta)]^{2}}{1+5 \alpha+18 \alpha \beta} \leq \\
\mu \leq \frac{8 \alpha(1+2 \beta) \delta^{2}+\left(\delta^{2}+\delta\right)[1+\alpha(1+4 \beta)]^{2}}{1+5 \alpha+18 \alpha \beta} ; \\
\frac{4 \mu \delta^{2}}{[1+\alpha(1+4 \beta)]^{2}}-\frac{16 \alpha(1+2 \beta) \delta^{2}+2 \delta^{2}[1+\alpha(1+4 \beta)]^{2}}{[1+\alpha(1+4 \beta)]^{2}(1+5 \alpha+18 \alpha \beta)} \\
\mu \geq \frac{8 \alpha(1+2 \beta) \delta^{2}+\left(\delta^{2}+\delta\right)[1+\alpha(1+4 \beta)]^{2}}{1+5 \alpha+18 \alpha \beta} .
\end{array}\right.
$$

PROOF:

By definition of $T K[\alpha, \beta, \delta]$,

$$
(1-\alpha) \frac{f(z)}{z}+\alpha \frac{\left\{z f^{\prime}(z)+\beta z^{2} f^{\prime \prime}(z)\right\}^{\prime}}{\{f(z)\}^{\prime}}=\left(\frac{1+w(z)}{1-w(z)}\right)^{\delta}
$$

where

$$
\begin{gathered}
w(z)=c_{1} z+c_{2} z^{2}+c_{3} z^{3}+ \\
f(z)=z+a_{2} z^{2}+a_{3} z^{3}+ \\
f^{\prime}(z)=1+2 a_{2} z+3 a_{3} z^{2}+4 a_{4} z^{3} \\
f^{\prime \prime}(z)=2 a_{2}+6 a_{3} z+12 a_{4} z^{2}
\end{gathered}
$$

Now by putting all these values in (6), we get: 


$$
(1-\alpha)\left(1+a_{2} z+a_{3} z^{2}+-\right)+\alpha\left(\frac{\left\{z+2 a_{2} z^{2}+3 a_{3} z^{3}+\beta 2 a_{2} z^{2}+\beta 6 a_{3} z^{3}+\beta 12 a_{4} z^{4}+\right\}^{\prime}}{\left(1+2 a_{2} z+3 a_{3} z^{2}+\right)}\right)=\left(\frac{1+c_{1} z+c_{2} z^{2}+---}{1-\left(c_{1} z+c_{2} z^{2}+---\right)}\right)^{\delta}
$$

By expanding the series, we get:

$$
1+[1+\alpha(1+4 \beta)] a_{2} z+\left[\{1+\alpha(5+18 \beta)\} a_{3}-4 \alpha(1+2 \beta) a_{2}^{2}\right] z^{2}+=1+2 \delta c_{1} z+2\left(\delta c_{2}+\delta^{2} c_{1}^{2}\right) z^{2}+
$$

By comparing, we get:

$$
a_{2}=\frac{2 \delta c_{1}}{1+\alpha(1+4 \beta)}
$$

and

$$
a_{3}=\frac{2[1+\alpha(1+4 \beta)]^{2} \delta c_{2}+\left[16 \alpha(1+2 \beta)+2\{1+\alpha(1+4 \beta)\}^{2}\right] \delta^{2} c_{1}^{2}}{\{1+\alpha(5+18 \beta)\}\{1+\alpha(1+4 \beta)\}^{2}}
$$

Using these values of $a_{2}$ and $a_{3}$, we get:

$$
a_{3}-\mu a_{2}^{2}=\frac{2 \delta c_{2}}{\{1+\alpha(5+18 \beta)\}}+\left(\frac{16 \alpha(1+2 \beta)+2\{1+\alpha(1+4 \beta)\}^{2}}{\{1+\alpha(5+18 \beta)\}\left(\{1+\alpha(1+4 \beta)\}^{2}\right)}-\frac{4 \mu}{\{1+\alpha(1+4 \beta)\}^{2}}\right) \delta^{2} c_{1}^{2}
$$

After applying mode on both sides, we get:

$$
\left|a_{3}-\mu a_{2}^{2}\right| \leq\left(\frac{2 \delta}{\{1+\alpha(5+18 \beta)\}}\right)\left|c_{2}\right|+\left|\left(\frac{16 \alpha(1+2 \beta)+2\{1+\alpha(1+4 \beta)\}^{2}}{\{1+\alpha(5+18 \beta)\}\{1+\alpha(1+4 \beta)\}^{2}}-\frac{4 \mu}{\{1+\alpha(1+4 \beta)\}^{2}}\right) \delta^{2}\right|\left|c_{1}\right|^{2}
$$

Using $\left|c_{2}\right| \leq 1-\left|c_{1}\right|^{2}$, we get:

$$
\left|a_{3}-\mu a_{2}^{2}\right| \leq \frac{2 \delta}{\{1+\alpha(5+18 \beta)\}}+\left\{\left|\left(\frac{16 \alpha(1+2 \beta)+2\{1+\alpha(1+4 \beta)\}^{2}}{\{1+\alpha(5+18 \beta)\}\{1+\alpha(1+4 \beta)\}^{2}}-\frac{4 \mu}{\{1+\alpha(1+4 \beta)\}^{2}}\right) \delta^{2}\right|-\frac{2 \delta}{\{1+\alpha(5+18 \beta)\}}\right\}\left|c_{1}\right|^{2}
$$

Case 1:

If $\mu \leq \frac{8 \alpha(1+2 \beta)+\{1+\alpha(1+4 \beta)\}^{2}}{2\{1+\alpha(5+18 \beta)\}}$, then,

$$
\left|a_{3}-\mu a_{2}^{2}\right| \leq \frac{2 \delta}{\{1+\alpha(5+18 \beta)\}}+\left\{\frac{16 \alpha(1+2 \beta) \delta^{2}+2\left(\delta^{2}-\delta\right)\{1+\alpha(1+4 \beta)\}^{2}}{\{1+\alpha(5+18 \beta)\}\{1+\alpha(1+4 \beta)\}^{2}}-\frac{4 \mu \delta^{2}}{\{1+\alpha(1+4 \beta)\}^{2}}\right\}\left|c_{1}\right|^{2}
$$

Subcase - 1 (a):

When

$$
\mu \leq \frac{8 \alpha(1+2 \beta) \delta^{2}+\left(\delta^{2}-\delta\right)\{1+\alpha(1+4 \beta)\}^{2}}{2 \delta^{2}\{1+\alpha(5+18 \beta)\}}
$$

Then, by using $\left|c_{1}\right| \leq 1$, we get:

$$
\left|a_{3}-\mu a_{2}^{2}\right| \leq \frac{2 \delta^{2}\{1+\alpha(1+4 \beta)\}^{2}+16 \alpha(1+2 \beta) \delta^{2}}{\{1+\alpha(5+18 \beta)\}\{1+\alpha(1+4 \beta)\}^{2}}-\frac{4 \mu \delta^{2}}{\{1+\alpha(1+4 \beta)\}^{2}}
$$

Subcase - 1 (b):

When $\mu \geq \frac{8 \alpha(1+2 \beta) \delta^{2}+\left(\delta^{2}-\delta\right)\{1+\alpha(1+4 \beta)\}^{2}}{2 \delta^{2}\{1+\alpha(5+18 \beta)\}}$, then,

$$
\left|a_{3}-\mu a_{2}^{2}\right| \leq \frac{2 \delta}{\{1+\alpha(5+18 \beta)\}}
$$

Case - 2:

If $\mu \geq \frac{8 \alpha(1+2 \beta)+\{1+\alpha(1+4 \beta)\}^{2}}{2\{1+\alpha(5+18 \beta)\}}$, then,

$$
\left|a_{3}-\mu a_{2}^{2}\right| \leq \frac{2 \delta}{\{1+\alpha(5+18 \beta)\}}+\left\{\frac{4 \mu \delta^{2}}{\{1+\alpha(1+4 \beta)\}^{2}}-\frac{16 \alpha(1+2 \beta) \delta^{2}+2\left(\delta^{2}+\delta\right)\{1+\alpha(1+4 \beta)\}^{2}}{\{1+\alpha(5+18 \beta)\}\{1+\alpha(1+4 \beta)\}^{2}}\right\}\left|c_{1}\right|^{2}
$$


Subcase-2 (a):

When $\mu \geq \frac{8 \alpha(1+2 \beta) \delta^{2}+\left(\delta^{2}+\delta\right)\{1+\alpha(1+4 \beta)\}^{2}}{2 \delta^{2}\{1+\alpha(5+18 \beta)\}}$, then,

$$
\left|a_{3}-\mu a_{2}^{2}\right| \leq-\frac{2 \delta^{2}\{1+\alpha(1+4 \beta)\}^{2}+16 \alpha(1+2 \beta) \delta^{2}}{\{1+\alpha(5+18 \beta)\}\{1+\alpha(1+4 \beta)\}^{2}}+\frac{4 \mu \delta^{2}}{\{1+\alpha(1+4 \beta)\}^{2}}
$$

Subcase - 2 (b):

When $\mu \leq \frac{8 \alpha(1+2 \beta) \delta^{2}+\left(\delta^{2}+\delta\right)\{1+\alpha(1+4 \beta)\}^{2}}{2 \delta^{2}\{1+\alpha(5+18 \beta)\}}$, then,

$$
\left|a_{3}-\mu a_{2}^{2}\right| \leq \frac{2 \delta}{\{1+\alpha(5+18 \beta)\}}
$$

Combining (7), (8), (9) and (10), we get the required result.

\section{EXTREMALS:}

The result is sharp for extremal functions:

$$
f(z)=z\left(1-\frac{2 \delta \alpha[(\alpha+5)+2 \beta\{3+4 \alpha(1+2 \beta)\}]}{[1+\alpha(1+4 \beta)][1+(5+18 \beta) \alpha]} z\right)^{-\frac{1+5 \alpha+18 \alpha \beta}{\alpha[(5+\alpha)+2 \beta\{3+4 \alpha(1+2 \beta)\}]}}
$$

and $f(z)=z\left(1+2 \delta z^{2}\right)^{\frac{1}{1+\alpha(5+18 \beta)}}$.

COROLLARY-4:

$T K[\alpha, \beta, 1]=T K[\alpha, \beta]$, as by substituting $\delta=1$, the result becomes:

$$
\left|a_{3}-\mu a_{2}^{2}\right| \leq\left\{\begin{array}{c}
\frac{16 \alpha(1+2 \beta)+2[1+\alpha(1+4 \beta)]^{2}}{[1+\alpha(1+4 \beta)]^{2}[1+(5+18 \beta) \alpha]}-\frac{4 \mu}{[1+\alpha(1+4 \beta)]^{2}} \\
\mu \leq \frac{4 \alpha(1+2 \beta)}{1+\alpha(5+18 \beta)} ; \\
\frac{2}{1+5 \alpha+18 \alpha \beta} ; \\
\mu \leq \frac{4 \alpha(1+2 \beta)+[1+\alpha(1+4 \beta)]^{2}}{1+\alpha(5+18 \beta)}
\end{array} ;\right.
$$

which is the required result for the class $T K[\alpha, \beta]$.

COROLLARY-5:

$T K[1,0,1]=K$, as by substituting $\alpha=1, \beta=0$ and $\delta=1$, the result becomes:

$$
\left|a_{3}-\mu a_{2}^{2}\right| \leq\left\{\begin{array}{l}
1-\mu, \text { if } \mu \leq \frac{2}{3} \\
\frac{1}{3}, \text { if } \frac{2}{3} \leq \mu \leq \frac{4}{3} \\
\mu-1, \text { if } \mu \geq \frac{4}{3}
\end{array}\right.
$$

which is the required result for the class $K$.

\section{THEOREM6:}

Let $f(z) \in T K[\alpha, \beta, A, B]$ and $\phi(z)=\frac{1+A w(z)}{1+B w(z)}$; $w(z)$ is a Schwarzian function, then: 


$$
\left|a_{3}-\mu a_{2}^{2}\right| \leq\left\{\begin{array}{c}
\frac{4 \alpha(1+2 \beta)(A-B)^{2}-B(A-B)[1+\alpha(1+4 \beta)]^{2}}{(1+5 \alpha+18 \alpha \beta)[1+\alpha(1+4 \beta)]^{2}}-\frac{\mu(A-B)^{2}}{[1+\alpha(1+4 \beta)]^{2}} \\
\mu \leq \frac{4 \alpha(1+2 \beta)(A-B)-(B+1)[1+\alpha(1+4 \beta)]^{2}}{(A-B)(1+5 \alpha+18 \alpha \beta)} \\
\frac{A-B}{1+5 \alpha+18 \alpha \beta}-\frac{4 \alpha(1+2 \beta)(A-B)-(B+1)[1+\alpha(1+4 \beta)]^{2}}{(A-B)(1+5 \alpha+18 \alpha \beta)} \leq \mu \\
\leq \frac{4 \alpha(1+2 \beta)(A-B)+(1-B)[1+\alpha(1+4 \beta)]^{2}}{(A-B)(1+5 \alpha+18 \alpha \beta)} \\
\frac{\mu(A-B)^{2}}{[1+\alpha(1+4 \beta)]^{2}}-\frac{4 \alpha(1+2 \beta)(A-B)^{2}-B(A-B)[1+\alpha(1+4 \beta)]^{2}}{(1+5 \alpha+18 \alpha \beta)[1+\alpha(1+4 \beta)]^{2}} \\
\mu \geq \frac{4 \alpha(1+2 \beta)(A-B)+(1-B)[1+\alpha(1+4 \beta)]^{2}}{(A-B)(1+5 \alpha+18 \alpha \beta)}
\end{array}\right.
$$

PROOF:

By definition of $T K[\alpha, \beta, A, B]$,

$$
(1-\alpha) \frac{f(z)}{z}+\alpha \frac{\left\{z f^{\prime}(z)+\beta z^{2} f^{\prime \prime}(z)\right\}^{\prime}}{\{f(z)\}^{\prime}}=\frac{1+A w(z)}{1+B w(z)}
$$

where

$$
\begin{gathered}
w(z)=c_{1} z+c_{2} z^{2}+c_{3} z^{3} \\
f(z)=z+a_{2} z^{2}+a_{3} z^{3} \\
f^{\prime}(z)=1+2 a_{2} z+3 a_{3} z^{2}+4 a_{4} z^{3} \\
f^{\prime \prime}(z)=2 a_{2}+6 a_{3} z+12 a_{4} z^{2}
\end{gathered}
$$

Now by putting all these values in (11), we get:

$$
(1-\alpha)\left(1+a_{2} z+a_{3} z^{2}-\right)+\alpha\left(\frac{\left\{z+2 a_{2} z^{2}+3 a_{3} z^{3}+\beta 2 a_{2} z^{2}+\beta 6 a_{3} z^{3}+\beta 12 a_{4} z^{4}+--------\right\}^{\prime}}{\left(1+2 a_{2} z+3 a_{3} z^{2}+----\right)}\right)=\frac{1+A\left(c_{1} z+c_{2} z^{2}+---\right)}{1+B\left(c_{1} z+c_{2} z^{2}+---\right)}
$$

By expanding the series, we get:

$$
\begin{gathered}
1+[1+\alpha(1+4 \beta)] a_{2} Z+\left[\{1+\alpha(5+18 \beta)\} a_{3}-4 \alpha(1+2 \beta) a_{2}^{2}\right] z^{2}=1+(A-B) c_{1} z+\left[(A-B) c_{2}-B(A-\right. \\
\left.B) c_{1}^{2}\right) z^{2}
\end{gathered}
$$

By comparing, we get:

$$
a_{2}=\frac{(A-B) c_{1}}{1+\alpha(1+4 \beta)}
$$

and

$$
a_{3}=\frac{(A-B)[1+\alpha(1+4 \beta)]^{2} c_{2}+(A-B)\left[4 \alpha(1+2 \beta)(A-B)-B\{1+\alpha(1+4 \beta)\}^{2}\right] c_{1}^{2}}{\{1+\alpha(5+18 \beta)\}\{1+\alpha(1+4 \beta)\}^{2}}
$$

Using these values of $a_{2}$ and $a_{3}$, we get:

$$
a_{3}-\mu a_{2}^{2}=\frac{(A-B) c_{2}}{1+\alpha(5+18 \beta)}+\left(\frac{\left[4 \alpha(1+2 \beta)(A-B)-B\{1+\alpha(1+4 \beta)\}^{2}\right](A-B)}{\{1+\alpha(5+18 \beta)\}\{1+\alpha(1+4 \beta)\}^{2}}-\frac{(A-B)^{2} \mu}{\{1+\alpha(1+4 \beta)\}^{2}}\right) c_{1}^{2}
$$

After applying mode on both sides, we get:

$$
\left|a_{3}-\mu a_{2}^{2}\right| \leq\left(\frac{(A-B)}{1+\alpha(5+18 \beta)}\right)\left|c_{2}\right|+\left|\frac{\left[4 \alpha(1+2 \beta)(A-B)-B\{1+\alpha(1+4 \beta)\}^{2}\right](A-B)}{\{1+\alpha(5+18 \beta)\}\{1+\alpha(1+4 \beta)\}^{2}}-\frac{(A-B)^{2} \mu}{\{1+\alpha(1+4 \beta)\}^{2}}\right|\left|c_{1}\right|^{2}
$$


Using $\left|c_{2}\right| \leq 1-\left|c_{1}\right|^{2}$, we get:

$$
\left|a_{3}-\mu a_{2}^{2}\right| \leq \frac{(A-B)}{1+\alpha(5+18 \beta)}+\left\{\left.\left|\left[\frac{\left[4 \alpha(1+2 \beta)(A-B)-B\{1+\alpha(1+4 \beta)\}^{2}\right](A-B)}{\{1+\alpha(5+18 \beta)\}\{1+\alpha(1+4 \beta)\}^{2}}-\frac{(A-B)^{2} \mu}{\{1+\alpha(1+4 \beta)\}^{2}} \mid-\frac{(A-B)}{1+\alpha(5+18 \beta)}\right\}\right| C_{1}\right|^{2}\right.
$$

Case 1:

If $\mu \leq \frac{4 \alpha(1+2 \beta)(A-B)-B\{1+\alpha(1+4 \beta)\}^{2}}{(A-B)\{1+\alpha(5+18 \beta)\}}$, then,

$$
\left|a_{3}-\mu a_{2}^{2}\right| \leq \frac{(A-B)}{1+\alpha(5+18 \beta)}+\left\{\frac{\left[4 \alpha(1+2 \beta)(A-B)-(B+1)\{1+\alpha(1+4 \beta)\}^{2}\right](A-B)}{\{1+\alpha(5+18 \beta)\}\{1+\alpha(1+4 \beta)\}^{2}}-\frac{(A-B)^{2} \mu}{\{1+\alpha(1+4 \beta)\}^{2}}\right\}\left|C_{1}\right|^{2}
$$

Subcase - 1 (a):

When $\mu \leq \frac{4 \alpha(1+2 \beta)(A-B)-(B+1)\{1+\alpha(1+4 \beta)\}^{2}}{(A-B)\{1+\alpha(5+18 \beta)\}}$

By using $\left|c_{1}\right| \leq 1$, we get:

$$
\left|a_{3}-\mu a_{2}^{2}\right| \leq \frac{\left[4 \alpha(1+2 \beta)(A-B)-B\{1+\alpha(1+4 \beta)\}^{2}\right](A-B)}{\{1+\alpha(5+18 \beta)\}\{1+\alpha(1+4 \beta)\}^{2}}-\frac{(A-B)^{2} \mu}{\{1+\alpha(1+4 \beta)\}^{2}}
$$

Subcase - 1 (b):

When $\mu \geq \frac{4 \alpha(1+2 \beta)(A-B)-(B+1)\{1+\alpha(1+4 \beta)\}^{2}}{(A-B)\{1+\alpha(5+18 \beta)\}}$, then,

$$
\left|a_{3}-\mu a_{2}^{2}\right| \leq \frac{(A-B)}{1+\alpha(5+18 \beta)}
$$

Case - 2:

If $\mu \geq \frac{4 \alpha(1+2 \beta)(A-B)-B\{1+\alpha(1+4 \beta)\}^{2}}{(A-B)\{1+\alpha(5+18 \beta)\}}$, then,

$$
\left|a_{3}-\mu a_{2}^{2}\right| \leq \frac{(A-B)}{1+\alpha(5+18 \beta)}+\left\{\frac{(A-B)^{2} \mu}{\{1+\alpha(1+4 \beta)\}^{2}}-\frac{\left[4 \alpha(1+2 \beta)(A-B)+(1-B)\{1+\alpha(1+4 \beta)\}^{2}\right](A-B)}{\{1+\alpha(5+18 \beta)\}\{1+\alpha(1+4 \beta)\}^{2}}\right\}\left|c_{1}\right|^{2}
$$

Subcase-2 (a):

When $\mu \geq \frac{4 \alpha(1+2 \beta)(A-B)+(1-B)\{1+\alpha(1+4 \beta)\}^{2}}{(A-B)\{1+\alpha(5+18 \beta)\}}$, then,

$$
\left|a_{3}-\mu a_{2}^{2}\right| \leq \frac{(A-B)^{2} \mu}{\{1+\alpha(1+4 \beta)\}^{2}}-\frac{\left[4 \alpha(1+2 \beta)(A-B)-B\{1+\alpha(1+4 \beta)\}^{2}\right](A-B)}{\{1+\alpha(5+18 \beta)\}\{1+\alpha(1+4 \beta)\}^{2}}
$$

Subcase - 2 (b):

When $\mu \leq \frac{4 \alpha(1+2 \beta)(A-B)+(1-B)\{1+\alpha(1+4 \beta)\}^{2}}{(A-B)\{1+\alpha(5+18 \beta)\}}$, then,

$$
\left|a_{3}-\mu a_{2}^{2}\right| \leq \frac{(A-B)}{1+\alpha(5+18 \beta)}
$$

Combining (12), (13), (14) and (15), we get the required result.

\section{EXTREMALS:}

Extremal functions of this inequality is given by:

$$
f(z)=\mathrm{Z}\left(1+\frac{2 \alpha \beta[A+B\{7+8 \alpha(1+2 \beta)\}]}{\{1+\alpha(5+18 \beta)\}\{1+\alpha(1+4 \beta)\}} Z\right)^{\frac{(1+5 \alpha+18 \alpha \beta)(A-B)}{A(1-3 \alpha)+B\left(1+2 \alpha^{2}+7 \alpha\right)+2 \alpha \beta[A+B\{7+8 \alpha(1+2 \beta)\}]}}
$$

and $f(z)=\mathrm{z}\left[1+(A-B) z^{2}\right]^{\frac{1}{1+\alpha(5+18 \beta)}}$.

COROLLARY-7:

$T K[\alpha, \beta, 1,-1]=T K[\alpha, \beta]$, as by substituting $\mathrm{A}=1$ and $\mathrm{B}=-1$, the result becomes: 


$$
\left|a_{3}-\mu a_{2}^{2}\right| \leq\left\{\begin{array}{c}
\frac{16 \alpha(1+2 \beta)+2[1+\alpha(1+4 \beta)]^{2}}{[1+\alpha(1+4 \beta)]^{2}(1+5 \alpha+18 \alpha \beta)}-\frac{4 \mu}{[1+\alpha(1+4 \beta)]^{2}} ; \mu \leq \frac{4 \alpha(1+2 \beta)}{1+5 \alpha+18 \alpha \beta} \\
\frac{2}{1+5 \alpha+18 \alpha \beta} ; \frac{4 \alpha(1+2 \beta)}{1+5 \alpha+18 \alpha \beta} \leq \mu \leq \frac{4 \alpha(1+2 \beta)+[1+\alpha(1+4 \beta)]^{2}}{(1+5 \alpha+18 \alpha \beta)}
\end{array}\right.
$$

which is the required result for the class $T K[\alpha, \beta]$.

\section{COROLLARY-8:}

$T K[1,0,1,-1]=K$, as by substituting $\alpha=1, \beta=0, \mathrm{~A}=1$ and $\mathrm{B}=-1$, the result becomes:

which is the required result for the class $K$.

$$
\left|a_{3}-\mu a_{2}^{2}\right| \leq\left\{\begin{array}{l}
1-\mu, \text { if } \mu \leq \frac{2}{3} \\
\frac{1}{3}, \text { if } \frac{2}{3} \leq \mu \leq \frac{4}{3} \\
\mu-1, \text { if } \mu \geq \frac{4}{3}
\end{array}\right.
$$

\section{THEOREM9:}

If $f(z) \in T K[\alpha, \beta, A, B, \delta]$ and $\phi(z)=\left(\frac{1+A w(z)}{1+B w(z)}\right)^{\delta}$, then:

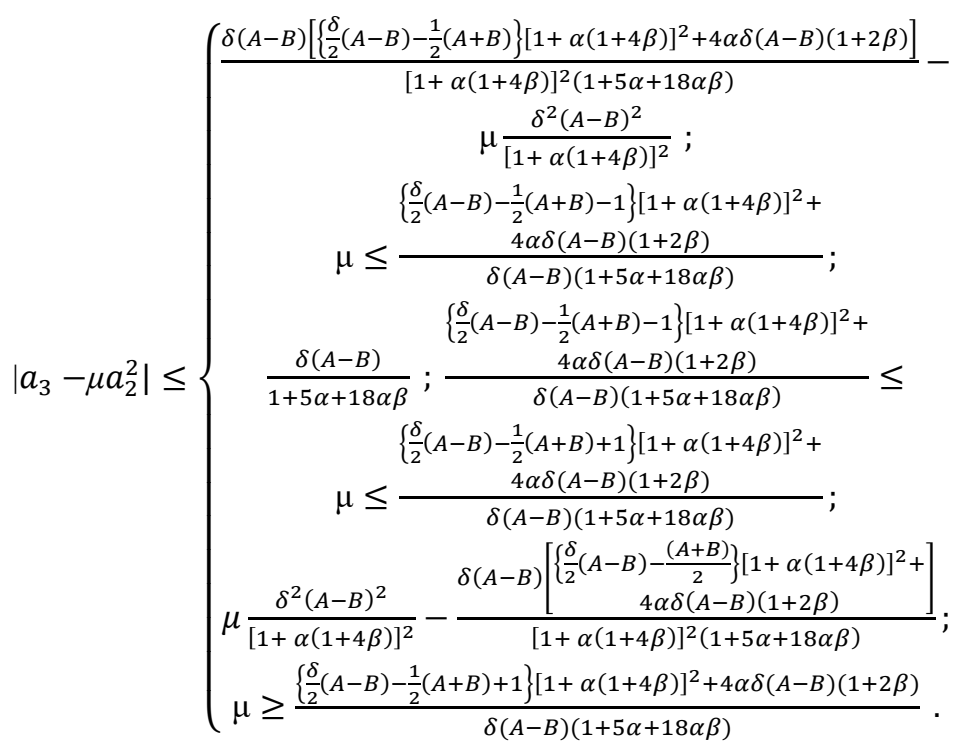

\section{PROOF:}

By definition of $T K[\alpha, \beta, A, B, \delta]$,

$$
(1-\alpha) \frac{f(z)}{z}+\alpha \frac{\left\{z f^{\prime}(z)+\beta z^{2} f^{\prime \prime}(z)\right\}^{\prime}}{\{f(z)\}^{\prime}}=\left(\frac{1+A w(z)}{1+B w(z)}\right)^{\delta}
$$

where

$$
\begin{gathered}
w(z)=c_{1} z+c_{2} z^{2}+c_{3} z^{3} \\
f(z)=z+a_{2} z^{2}+a_{3} z^{3} \\
f^{\prime}(z)=1+2 a_{2} z+3 a_{3} z^{2}+4 a_{4} z^{3} \\
f^{\prime \prime}(z)=2 a_{2}+6 a_{3} z+12 a_{4} z^{2}
\end{gathered}
$$

Now, by putting all these values in (16), we get: 


$$
\begin{aligned}
(1-\alpha)\left(1+a_{2} z\right. & \left.+a_{3} z^{2}+-\right)+\alpha\left(\frac{\left\{z+2 a_{2} z^{2}+3 a_{3} z^{3}+\beta 2 a_{2} z^{2}+\beta 6 a_{3} z^{3}+\beta 12 a_{4} z^{4}+\right\}^{\prime}}{\left(1+2 a_{2} z+3 a_{3} z^{2}+\right)}\right) \\
& =\left(\frac{1+A\left(c_{1} z+c_{2} z^{2}+\right)}{1+B\left(c_{1} z+c_{2} z^{2}+\right)}\right)^{\delta}
\end{aligned}
$$

By expanding the series, we get:

$$
\begin{gathered}
1+[1+\alpha(1+4 \beta)] a_{2} z+\left[\{1+\alpha(5+18 \beta)\} a_{3}-4 \alpha(1+2 \beta) a_{2}^{2}\right] z^{2} \\
=1+\delta(A-B) c_{1} z+\left[\delta(A-B) c_{2}+\frac{\delta^{2}}{2}\left(A^{2}+B^{2}-2 A B\right) c_{1}^{2}-\frac{\delta}{2}\left(A^{2}-B^{2}\right) c_{1}^{2}\right] z^{2}-
\end{gathered}
$$

By comparing, we get:

$$
a_{2}=\frac{\delta(A-B) c_{1}}{1+\alpha(1+4 \beta)}
$$

and

$$
a_{3}=\frac{\delta(A-B)[1+\alpha(1+4 \beta)]^{2} c_{2}+\delta(A-B)[4 \alpha \delta(1+2 \beta)(A-B)+}{\left.\frac{1}{2}\{\delta(A-B)-(A+B)\}\{1+\alpha(1+4 \beta)\}^{2}\right] c_{1}^{2}}
$$

Using these values of $a_{2}$ and $a_{3}$, we get:

$$
a_{3}-\mu a_{2}^{2}=\frac{\delta(A-B) c_{2}}{1+\alpha(5+18 \beta)}+\left(\frac{\delta(A-B)\left[4 \alpha \delta(1+2 \beta)(A-B)+\frac{1}{2}\{\delta(A-B)-(A+B)\}\{1+\alpha(1+4 \beta)\}^{2}\right]}{\{1+\alpha(5+18 \beta)\}\{1+\alpha(1+4 \beta)\}^{2}}-\frac{\delta^{2}(A-B)^{2} \mu}{\{1+\alpha(1+4 \beta)\}^{2}}\right) c_{1}^{2}
$$

After applying mode on both sides,

we get

$$
\left|a_{3}-\mu a_{2}^{2}\right| \leq\left(\frac{\delta(A-B)}{1+\alpha(5+18 \beta)}\right)\left|c_{2}\right|+\left|\frac{\delta(A-B)\left[4 \alpha \delta(1+2 \beta)(A-B)+\frac{1}{2}\{\delta(A-B)-(A+B)\}\{1+\alpha(1+4 \beta)\}^{2}\right]}{\{1+\alpha(5+18 \beta)\}\{1+\alpha(1+4 \beta)\}^{2}}-\frac{\delta^{2}(A-B)^{2} \mu}{\{1+\alpha(1+4 \beta)\}^{2}}\right|\left|C_{1}\right|^{2}
$$

Using $\left|c_{2}\right| \leq 1-\left|c_{1}\right|^{2}$, we get:

$$
\begin{gathered}
\left|a_{3}-\mu a_{2}^{2}\right| \leq \frac{\delta(A-B)}{1+\alpha(5+18 \beta)}+\left\{\left|\frac{\delta(A-B)\left[4 \alpha \delta(1+2 \beta)(A-B)+\frac{1}{2}\{\delta(A-B)-(A+B)\}\{1+\alpha(1+4 \beta)\}^{2}\right]}{\{1+\alpha(5+18 \beta)\}\{1+\alpha(1+4 \beta)\}^{2}}-\frac{\delta^{2}(A-B)^{2} \mu}{\{1+\alpha(1+4 \beta)\}^{2}}\right|-\right. \\
\left.\frac{\delta(A-B)}{1+\alpha(5+18 \beta)}\right\}\left|C_{1}\right|^{2}
\end{gathered}
$$

\section{Case 1:}

If $\mu \leq \frac{\left[4 \alpha \delta(1+2 \beta)(A-B)+\frac{1}{2}\{\delta(A-B)-(A+B)\}\{1+\alpha(1+4 \beta)\}^{2}\right]}{\delta(A-B)\{1+\alpha(5+18 \beta)\}}$, then,

$$
\left|a_{3}-\mu a_{2}^{2}\right| \leq \frac{\delta(A-B)}{1+\alpha(5+18 \beta)}+\left\{\frac{\delta(A-B)\left[4 \alpha \delta(1+2 \beta)(A-B)+\left\{\frac{\delta}{2}(A-B)-\frac{1}{2}(A+B)-1\right\}\{1+\alpha(1+4 \beta)\}^{2}\right]}{\{1+\alpha(5+18 \beta)\}\{1+\alpha(1+4 \beta)\}^{2}}-\frac{\delta^{2}(A-B)^{2} \mu}{\{1+\alpha(1+4 \beta)\}^{2}}\right\}\left|c_{1}\right|^{2}
$$

\section{Subcase - 1 (a):}

When $\mu \leq \frac{4 \alpha \delta(1+2 \beta)(A-B)+\left\{\frac{\delta}{2}(A-B)-\frac{1}{2}(A+B)-1\right\}\{1+\alpha(1+4 \beta)\}^{2}}{\delta(A-B)\{1+\alpha(5+18 \beta)\}}$, then, by using $\left|C_{1}\right| \leq 1$, we get:

$$
\left|a_{3}-\mu a_{2}^{2}\right| \leq \frac{\delta(A-B)\left[4 \alpha \delta(1+2 \beta)(A-B)+\left\{\frac{\delta}{2}(A-B)-\frac{1}{2}(A+B)\right\}\{1+\alpha(1+4 \beta)\}^{2}\right]}{\{1+\alpha(5+18 \beta)\}\{1+\alpha(1+4 \beta)\}^{2}}-\frac{\delta^{2}(A-B)^{2} \mu}{\{1+\alpha(1+4 \beta)\}^{2}}
$$


Subcase - 1 (b):

When $\mu \geq \frac{4 \alpha \delta(1+2 \beta)(A-B)+\left\{\frac{\delta}{2}(A-B)-\frac{1}{2}(A+B)-1\right\}\{1+\alpha(1+4 \beta)\}^{2}}{\delta(A-B)\{1+\alpha(5+18 \beta)\}}$, then,

$$
\left|a_{3}-\mu a_{2}^{2}\right| \leq \frac{\delta(A-B)}{1+\alpha(5+18 \beta)}
$$

Case - 2:

If $\mu \geq \frac{\left[4 \alpha \delta(1+2 \beta)(A-B)+\frac{1}{2}\{\delta(A-B)-(A+B)\}\{1+\alpha(1+4 \beta)\}^{2}\right]}{\delta(A-B)\{1+\alpha(5+18 \beta)\}}$, then,

$$
\left|a_{3}-\mu a_{2}^{2}\right| \leq \frac{\delta(A-B)}{1+\alpha(5+18 \beta)}+\left\{\frac{\delta^{2}(A-B)^{2} \mu}{\{1+\alpha(1+4 \beta)\}^{2}}-\frac{\delta(A-B)\left[4 \alpha \delta(1+2 \beta)(A-B)+\left\{\frac{\delta}{2}(A-B)-\frac{1}{2}(A+B)+1\right\}\{1+\alpha(1+4 \beta)\}^{2}\right]}{\{1+\alpha(5+18 \beta)\}\{1+\alpha(1+4 \beta)\}^{2}}\right\}\left|c_{1}\right|^{2}
$$

Subcase-2 (a):

When $\mu \geq \frac{4 \alpha \delta(1+2 \beta)(A-B)+\left\{\frac{\delta}{2}(A-B)-\frac{1}{2}(A+B)+1\right\}\{1+\alpha(1+4 \beta)\}^{2}}{\delta(A-B)\{1+\alpha(5+18 \beta)\}}$, then,

$\left|a_{3}-\mu a_{2}^{2}\right| \leq \frac{\delta^{2}(A-B)^{2} \mu}{\{1+\alpha(1+4 \beta)\}^{2}}-\frac{\delta(A-B)\left[4 \alpha \delta(1+2 \beta)(A-B)+\left\{\frac{\delta}{2}(A-B)-\frac{1}{2}(A+B)\right\}\{1+\alpha(1+4 \beta)\}^{2}\right]}{\{1+\alpha(5+18 \beta)\}\{1+\alpha(1+4 \beta)\}^{2}}$

Subcase -2 (b):

When $\mu \leq \frac{4 \alpha \delta(1+2 \beta)(A-B)+\left\{\frac{\delta}{2}(A-B)-\frac{1}{2}(A+B)+1\right\}\{1+\alpha(1+4 \beta)\}^{2}}{\delta(A-B)\{1+\alpha(5+18 \beta)\}}$, then,

$$
\left|a_{3}-\mu a_{2}^{2}\right| \leq \frac{\delta(A-B)}{1+\alpha(5+18 \beta)}
$$

By combining (17), (18), (19) and (20), we get the required result.

\section{FOR EXTREMALS:}

Extremal functions of this inequality is given by:

$$
f(z)=z\left(\begin{array}{c}
\delta(A-B)(1-3 \alpha+2 \alpha \beta) \\
-[1+\alpha(1+4 \beta)]^{2} \\
1+\frac{\delta(A-B)-(A+B)\}}{(1+5 \alpha+18 \alpha \beta)[1+\alpha(1+4 \beta)]^{2}} z
\end{array}\right)^{\frac{\delta(A-B)(1+5 \alpha+18 \alpha \beta)}{\delta(A-B)(1-3 \alpha+2 \alpha \beta)[1+\alpha(1+4 \beta)]^{2}-\{\delta(A-B)-(A+B)\}}}
$$

and $f(z)=z\left[1+\delta(A-B) z^{2}\right]^{\frac{1}{1+5 \alpha+18 \alpha \beta}}$.

COROLLARY-10:

$T K[\alpha, \beta, 1,-1,1]=T K[\alpha, \beta]$, as by substituting $A=1, B=-1$ and $\delta=1$, the result becomes:

$$
\left|a_{3}-\mu a_{2}^{2}\right| \leq\left\{\begin{array}{c}
\frac{16 \alpha(1+2 \beta)+2[1+\alpha(1+4 \beta)]^{2}}{[1+\alpha(1+4 \beta)]^{2}(1+5 \alpha+18 \alpha \beta)}-\frac{4 \mu}{[1+\alpha(1+4 \beta)]^{2}} ; \mu \leq \frac{4 \alpha(1+2 \beta)}{1+5 \alpha+18 \alpha \beta} \\
\frac{2}{1+5 \alpha+18 \alpha \beta} ; \frac{4 \alpha(1+2 \beta)}{1+5 \alpha+18 \alpha \beta} \leq \mu \leq \frac{4 \alpha(1+2 \beta)+[1+\alpha(1+4 \beta)]^{2}}{(1+5 \alpha+18 \alpha \beta)} \\
\frac{4 \mu}{[1+\alpha(1+4 \beta)]^{2}}-\frac{16 \alpha(1+2 \beta)+2[1+\alpha(1+4 \beta)]^{2}}{[1+\alpha(1+4 \beta)]^{2}(1+5 \alpha+18 \alpha \beta)} \\
\mu \geq \frac{4 \alpha(1+2 \beta)+[1+\alpha(1+4 \beta)]^{2}}{(1+5 \alpha+18 \alpha \beta)}
\end{array}\right.
$$

which is the required result for the class $\operatorname{TK}[\alpha, \beta]$.

COROLLARY-11:

$T K[\alpha, \beta, 1,-1, \delta]=T K[\alpha, \beta, \delta]$, as by substituting $\mathrm{A}=1$ and $\mathrm{B}=-1$, the result becomes: 
which is the required result for the class $T K[\alpha, \beta, \delta]$.

$$
\left|a_{3}-\mu a_{2}^{2}\right| \leq\left\{\begin{array}{c}
\frac{16 \alpha(1+2 \beta) \delta^{2}+2 \delta^{2}[1+\alpha(1+4 \beta)]^{2}}{[1+\alpha(1+4 \beta)]^{2}(1+5 \alpha+18 \alpha \beta)}-\frac{4 \mu \delta^{2}}{[1+\alpha(1+4 \beta)]^{2}} \\
\mu \leq \frac{8 \alpha(1+2 \beta) \delta^{2}+\left(\delta^{2}-\delta\right)[1+\alpha(1+4 \beta)]^{2}}{1+5 \alpha+18 \alpha \beta} ; \\
\frac{2 \delta}{1+5 \alpha+18 \alpha \beta} ; \frac{8 \alpha(1+2 \beta) \delta^{2}+\left(\delta^{2}-\delta\right)[1+\alpha(1+4 \beta)]^{2}}{1+5 \alpha+18 \alpha \beta} \leq \mu \\
\leq \frac{8 \alpha(1+2 \beta) \delta^{2}+\left(\delta^{2}+\delta\right)[1+\alpha(1+4 \beta)]^{2}}{1+5 \alpha+18 \alpha \beta} \\
\frac{4 \mu \delta^{2}}{[1+\alpha(1+4 \beta)]^{2}}-\frac{16 \alpha(1+2 \beta) \delta^{2}+2 \delta^{2}[1+\alpha(1+4 \beta)]^{2}}{[1+\alpha(1+4 \beta)]^{2}(1+5 \alpha+18 \alpha \beta)} \\
\mu \geq \frac{8 \alpha(1+2 \beta) \delta^{2}+\left(\delta^{2}+\delta\right)[1+\alpha(1+4 \beta)]^{2}}{1+5 \alpha+18 \alpha \beta}
\end{array}\right.
$$

COROLLARY-12:

$T K[\alpha, \beta, A, B, 1]=T K[\alpha, \beta, A, B]$, as by substituting $\delta=1$, the result becomes:

$$
\left|a_{3}-\mu a_{2}^{2}\right| \leq\left\{\begin{array}{c}
\frac{4 \alpha(1+2 \beta)(A-B)^{2}-B(A-B)[1+\alpha(1+4 \beta)]^{2}}{(1+5 \alpha+18 \alpha \beta)[1+\alpha(1+4 \beta)]^{2}}-\frac{\mu(A-B)^{2}}{[1+\alpha(1+4 \beta)]^{2}} \\
\mu \leq \frac{4 \alpha(1+2 \beta)(A-B)-(B+1)[1+\alpha(1+4 \beta)]^{2}}{(A-B)(1+5 \alpha+18 \alpha \beta)} ; \\
\frac{A-B}{1+5 \alpha+18 \alpha \beta} ; \frac{4 \alpha(1+2 \beta)(A-B)-(B+1)[1+\alpha(1+4 \beta)]^{2}}{(A-B)(1+5 \alpha+18 \alpha \beta)} \leq \\
\mu \leq \frac{4 \alpha(1+2 \beta)(A-B)+(1-B)[1+\alpha(1+4 \beta)]^{2}}{(A-B)(1+5 \alpha+18 \alpha \beta)} ; \\
\frac{\mu(A-B)^{2}}{[1+\alpha(1+4 \beta)]^{2}}-\frac{4 \alpha(1+2 \beta)(A-B)^{2}-B(A-B)[1+\alpha(1+4 \beta)]^{2}}{(1+5 \alpha+18 \alpha \beta)[1+\alpha(1+4 \beta)]^{2}} \\
\mu \geq \frac{4 \alpha(1+2 \beta)(A-B)+(1-B)[1+\alpha(1+4 \beta)]^{2}}{(A-B)(1+5 \alpha+18 \alpha \beta)}
\end{array}\right.
$$

which is the required result for the class $T K[\alpha, \beta, A, B]$.

\section{REFERENCES}

[1] De Branges, L. (1985), A proof of Bieberbach Conjecture, Acta. Math., vol. 154, pp. 137-152.

[2] Duren, P.L. (1977), Coefficient of univalent functions, Bulletin of American Mathematical Society, vol. 83, pp. 891-911.

[3] Fekete, M. and Szegö, G. (1933), Eine Bemerkung uber ungerade schlichte funktionen, J. London Math. Soc., vol. 8, pp. 85-89.

[4] Garabedian, P.R. and Schiffer, M. (1955), A Proof for the Bieberbach Conjecture for the fourth coefficient, Arch. Rational Mech. Anal., vol. 4, pp. 427-465.

[5] Keogh, F. R. and Merkes, E. P. (1989), A coefficient inequality for certain classes of analytic functions, Proc. Of Amer. Math. Soc., vol. 20, pp. 8-12.

[6] Koebe, P. (1907), Uber Die uniformisierung beliebiger analytischer Kurven, Nach. Ges. Wiss. Gottingen, vol. 1907, pp. 633669.

[7] Lindelof, E. (1909), Memoire sur certaines inegalities dans la theorie des functions monogenes et sur quelquesproprieties nouvellles de ces fonctions dans la voisinaged'unpointsingulier essential, Acta Soc. Sci. Fenn., vol. 23, pp. 481-519.

[8] Löewner, C. (1923), Untersuchungen uber schlichte konforme Abbildungen des Einheitskreises I, Math. Ann., vol. 89, pp. 103121.

[9] Miller, S.S., Mocanu, P.T. and Reade, M.O. (1973), All convex functions are univalent and starlike, Proceedings of American Mathematical Society, vol. 37, pp. 553-554.

[10] Minda, D. and MA, W., A unified treatment of some special classes of univalent functions, In proceedings of the conference on complex analysis, Z. Li, F. Ren, I. Yang and S. Zhang (Eds), Int. Press (1994), 157-169.

[11] Nevanlinna, R. (1922), Uber die Eigenschaften einer analytischen funktionen in der Umgebung einer singulären Stele Order Linie, Acta Soc. Sci. Fenn., vol. 50, pp. 1-46.

[12] Pederson, R. (1968-69), A proof for the Bieberbach conjecture for the sixth coefficient, Arch. Rational Mech. Anal., vol. 31, pp. 331-351.

[13] Pederson, R. and Schiffer, M. (1972), A proof for the Bieberbach conjecture for the fifth coefficient, Arch. Rational Mech. Anal., vol. 45, pp. 161-193.

[14] Singh, G. (2014), Fekete-Szego Inequality for a new class and its certain subclasses of analytic functions, General Mathematical Notes, vol. 21, pp. 86-96. 